\title{
Mid-infrared electric field characterization using a visible charge-coupled-device-based spectrometer
}

\author{
Kevin J. Kubarych and Manuel Joffre \\ Laboratoire d'Optique et Biosciences, Centre National de la Recherche Scientifique, Unite Mixte de Recherche 7645, \\ INSERM U 451, Ecole Polytechnique, Ecole Nationale Supérieure de Techniques Avanćees, \\ 91128 Palaiseau, France
}

Amy Moore, Nadia Belabas, and David M. Jonas

Department of Chemistry and Biochemistry, University of Colorado at Boulder, Boulder, Colorado 80309-0215

Received November 15, 2004

\begin{abstract}
We characterize ultrashort mid-infrared pulses through upconversion by using the stretched pulses obtained from the uncompressed output of a chirped-pulse amplifier. The power spectrum thus translated into the visible region can be readily measured with a standard silicon CCD camera-based spectrometer. The spectral phase is also characterized by a variant of zero-added-phase spectral phase interferometry for direct electric field reconstruction. This is a general method that provides a multiplex advantage over conventional infrared detector array-based methods. (C) 2005 Optical Society of America
\end{abstract}

OCIS codes: $320.7100,120.5050,120.6200$.

The IR spectral region is rich in chemical information relating directly to molecular structure and dynamics and benefits from being free of the complications of excited electronic state dynamics. Progress in ultrafast IR spectroscopy has recently begun a rapid acceleration largely because of the conceptual breakthrough of adopting two-dimensional (2D) techniques analogous to those used in nuclear magnetic resonance spectroscopy. Central to these techniques is the measurement of the field of the generated nonlinear optical signal. Although $2 \mathrm{D}$ spectroscopy can be readily implemented in the visible spectral range with high-resolution silicon CCD cameras, ${ }^{1-4}$ most advances in 2D IR spectroscopy have relied either on time-consuming scanning in either the frequency ${ }^{5}$ or time domains ${ }^{6,7}$ or on expensive HgCdTe detector arrays of $\sim 64$ pixels. ${ }^{8,9}$ Although progress may be expected in IR detector arrays, their multiplex advantage is limited by the IR diffraction limit.

In this Letter we report a novel approach to IR spectroscopy based on upconverting a mid-IR pulse with the uncompressed output of a Ti:sapphire chirped-pulse amplifier (CPA), effectively translating the IR spectrum into the visible. We note that the upconversion approach for detecting mid-IR spectra with a CCD camera has been reported previously, ${ }^{10}$ although it was based on the use of a narrowband visible pulse obtained by frequency doubling a picosecond mode-locked Nd:YAG laser. Highly stretched $800-\mathrm{nm}$ pulses are readily available from most Ti:sapphire CPA systems. Further, it is straightforward to determine the spectral phase as well as the spectral amplitude with a simple extension of this frequency conversion: By upconverting the IR test pulse with two time-delay replicas of the $800-\mathrm{nm}$ stretched pulse in a noncollinear geometry, two sheared pulses are generated in the visible $(\sim 700 \mathrm{~nm})$. Since this approach requires only one test pulse, it is possible to measure the pulse in situ, and thus it has been previously denoted zeroadditional-phase (ZAP) spectral phase interferometry for direct electric field reconstruction (SPIDER). ${ }^{11,12}$ It has been demonstrated to be a powerful variant of the SPIDER technique ${ }^{13}$ for the measurement of near-IR ${ }^{11}$ or UV pulses. ${ }^{12}$ It is particularly noteworthy that the results presented in this Letter used only $\sim 5 \mu \mathrm{J}$ of energy in each $800-\mathrm{nm}$ chirped pulse, derived from the zero-order diffraction from the compressor grating in a CPA system, thus yielding substantial linear chirp with no additional stretcher from a beam that is typically unused.

Figure 1 shows the experimental diagram for our apparatus. The distinguishing feature of ZAP SPIDER is that there is only a single test pulse and two replicas of the stretched pulse. In this case, the shear, $\Omega$, is determined by the time delay between the two stretched pulses, denoted $\tau_{\Omega}$. To introduce a

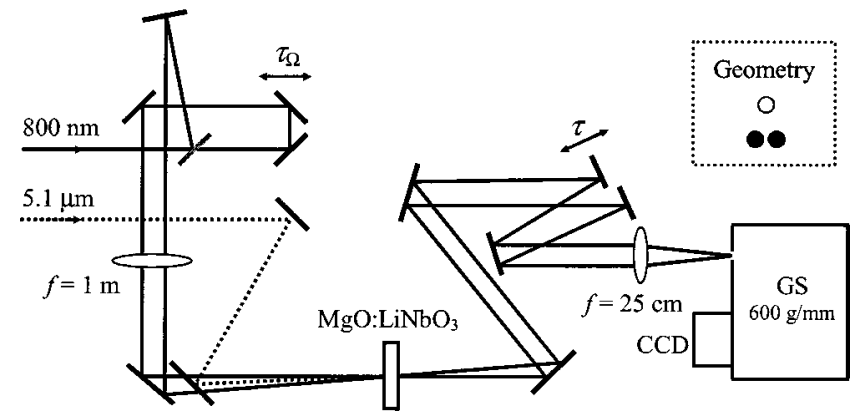

Fig. 1. Upconversion and ZAP SPIDER apparatus. Two chirped $800-\mathrm{nm}$ pulses $(5 \mu \mathrm{J}$ each, $150 \mathrm{ps})$ are derived from the zero-order diffraction of the CPA compressor and mixed noncollinearly with the $1-\mu \mathrm{J}$ mid-IR pulses in a 425- $\mu \mathrm{m} \mathrm{MgO}: \mathrm{LiNbO}_{3}$ crystal. The signals are imaged on the slit (set at $8 \mu \mathrm{m}$ ) of a grating spectrometer (GS). Inset, upconversion beam geometry of mid-IR (white) and stretched (black) pulses. 
time delay, $\tau$, between the two upconverted visible pulses, it is necessary to separate them spatially by using a slightly noncollinear beam geometry (inset of Fig. 1). The two sheared pulses are then imaged noncollinearly onto the entrance slit $(8 \mu \mathrm{m})$ of a monochromator. Selecting only the central fringe of the resulting spatial interference pattern $(25-\mu \mathrm{m}$ period) provides the spectral interference necessary for a SPIDER measurement. The spectrum was dispersed by the first order of a 600 -groove $/ \mathrm{mm}$ grating in a 0.46 - $m$ spectrometer (SPEX HR 460) and was recorded with a 25.4 -mm-wide $1340 \times 100$ pixel silicon CCD camera with 16 -bit, $2-\mathrm{MHz}$ analog-to-digital conversion (Roper Scientific Evry, France).

The mid-IR pulses were created with a $\beta$-barium borate, white-light-seeded optical parametric amplifier generating signal and idler beams that were subsequently difference frequency mixed in a $1-\mathrm{mm}$ GaSe crystal, resulting in $1-\mu \mathrm{J}, 5.1-\mu \mathrm{m}$ pulses. The $1 / e^{2}$ beam diameter of the mid-IR pulses was $1.5 \mathrm{~mm}$ at the $425-\mu \mathrm{m}$-thick $\mathrm{MgO}: \mathrm{LiNbO}_{3}\left(5 \% \mathrm{MgO}, \theta=46^{\circ}\right.$, type I) crystal. The $\sim 150$-ps stretched pulses were obtained from the zero-order diffraction in the compressor of our 1-kHz Ti:sapphire regenerative amplifier (Spectra-Physics Hurricane). In contrast to previous implementations of ZAP SPIDER-where the dispersion of a small amount of glass was used to stretch the pulses to only $2 \mathrm{ps}^{11,12}$ - the upconverted signal experiences negligible spectral broadening $\left(<0.3 \mathrm{~cm}^{-1}\right)$. A telescope (not shown) adapts the diameter of the stretched pulse to roughly $2 \mathrm{~mm}$, and a long-focal-length lens loosely focuses the two beams roughly $20 \mathrm{~cm}$ downstream of the upconversion crystal. With $5 \mu \mathrm{J}$ in each stretched pulse the upconversion signal was visible to the eye.

To determine the unknown spectral phase of the mid-IR pulse, the contribution of the delay between the two frequency-shifted pulses, $\omega \tau$, must be subtracted from the phase difference. ${ }^{13}$ This subtraction can be done by measuring a background spectral interferogram in the absence of any spectral shear, that is, with $\tau_{\Omega}=0$, and thus $\Omega=0$. By adjusting the time delay, $\tau_{\Omega}$, in the stretched pulse interferometer so that the two upconverted spectra are identical, the phase determined from this background interferogram is given only by time delay $\tau$ and can be subtracted from subsequent SPIDER phase measurements (with $\Omega \neq 0$ ). Since any change in $\tau_{\Omega}$ does not affect delay $\tau$, one is free to choose any spectral shear after having recorded the background interferogram.

One difficulty of ZAP SPIDER with a highly chirped mixing pulse is the determination of the absolute frequency of the mid-IR pulse. Knowledge of the upconverted signal frequency does not uniquely determine the IR frequency unless one has precise knowledge of which frequency from the chirped pulse coincided with the IR pulse. The most accurate determination is a reference-free method using timedomain interferometry (i.e., Fourier transform spectroscopy). For this Letter, however, we have determined the absolute mid-IR frequency by using a vibrational transition, that of carbon monoxide bound within the protein hemoglobin $(\mathrm{HbCO})$, since this transition is very narrow $\left(\sim 12 \mathrm{~cm}^{-1}\right)$ and its frequency is well known to be $1951 \mathrm{~cm}^{-1}{ }^{14}$ Figure 2 shows the upconverted spectrum after transmission of the mid-IR pulse through a 5 - $\mathrm{mM}$ sample of $\mathrm{HbCO}$ (optical density 0.18 at $1951 \mathrm{~cm}^{-1}$ ). ${ }^{14}$ The sample path length was $50 \mu \mathrm{m}$ with 2 -mm-thick $\mathrm{CaF}_{2}$ windows. The upper horizontal scale shows the frequency in wave numbers obtained by converting from the wavelength and subtracting a constant offset such that the $\mathrm{CO}$ vibration was centered at $1951 \mathrm{~cm}^{-1}$. All the spectra shown here were obtained with an exposure time of $1 \mathrm{~ms}$ and a total readout time for our laser-synchronized CCD system of $1.5 \mathrm{~ms}$. Although these measurements are not rigorously single shot, the measured signal levels indicate that single-shot operation is possible with faster detection.

The ZAP SPIDER trace of an unshaped mid-IR pulse is shown in Fig. 3(a). From the interferogram of the unsheared pulse (not shown), the delay between the two chirped pulses was determined to be $2.8 \mathrm{ps}$. The shear was set to be $1 \mathrm{THz}$. The observed fringe contrast of 0.6 results from a combination of imperfect alignment of the interferometer and finite slit width. The recovered amplitude and phase are shown in Fig. 3(b). As expected, ${ }^{15}$ the output of the difference-frequency generation is largely transform limited. The mid-IR pulses were shaped by using either a $6.3-\mathrm{cm}$ block of $\mathrm{CaF}_{2}$ or a $6-\mathrm{mm}$ plate of $\mathrm{Ge}-$ two oppositely dispersive materials. Figure 4 shows the recovered phase and amplitude of the shaped pulses traversing the different transparent media with the unshaped phase of Fig. 3 subtracted, yielding the phase introduced by the material. Comparison with the calculated phase shows excellent agreement. ${ }^{16}$ As a measure of the very high spectral resolution, only one quarter of the phase points are shown in Figs. 3 and 4 . There were typically 200 well-determined phase points in a spectrum with a pixel-size-limited mid-IR spectral resolution of $1.4 \mathrm{~cm}^{-1}(\Delta \lambda=0.07 \mathrm{~nm}$ at $693 \mathrm{~nm})$. This resolution compares favorably with that achievable with $\mathrm{HgCdTe}$ detector arrays. Moreover, since the camera

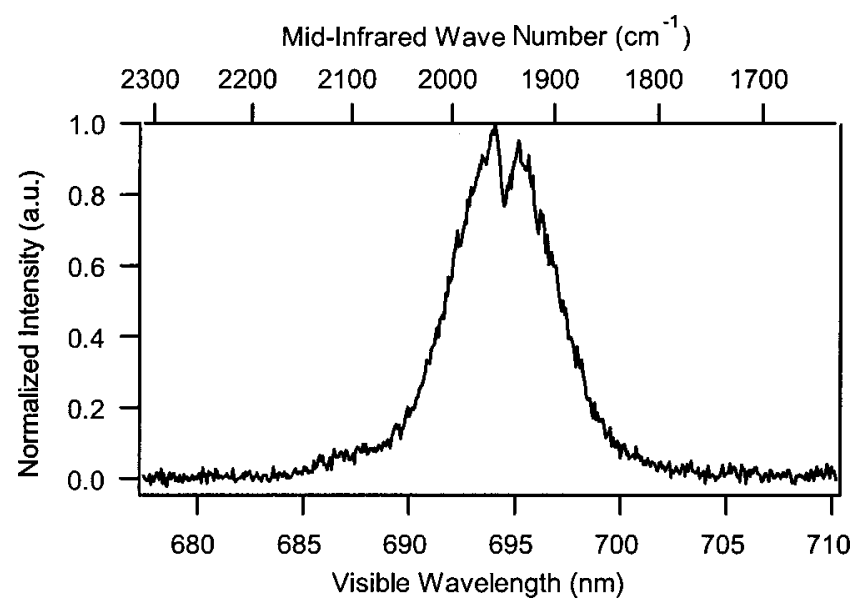

Fig. 2. Spectrum of mid-IR pulses after transmission through $5 \mathrm{mM}$ of a solution of $\mathrm{HbCO}$ in a $\mathrm{D}_{2} \mathrm{O}$ buffer solution. The frequency axis was shifted to the center of the peak of the absorption feature at $1951 \mathrm{~cm}^{-1}$. 


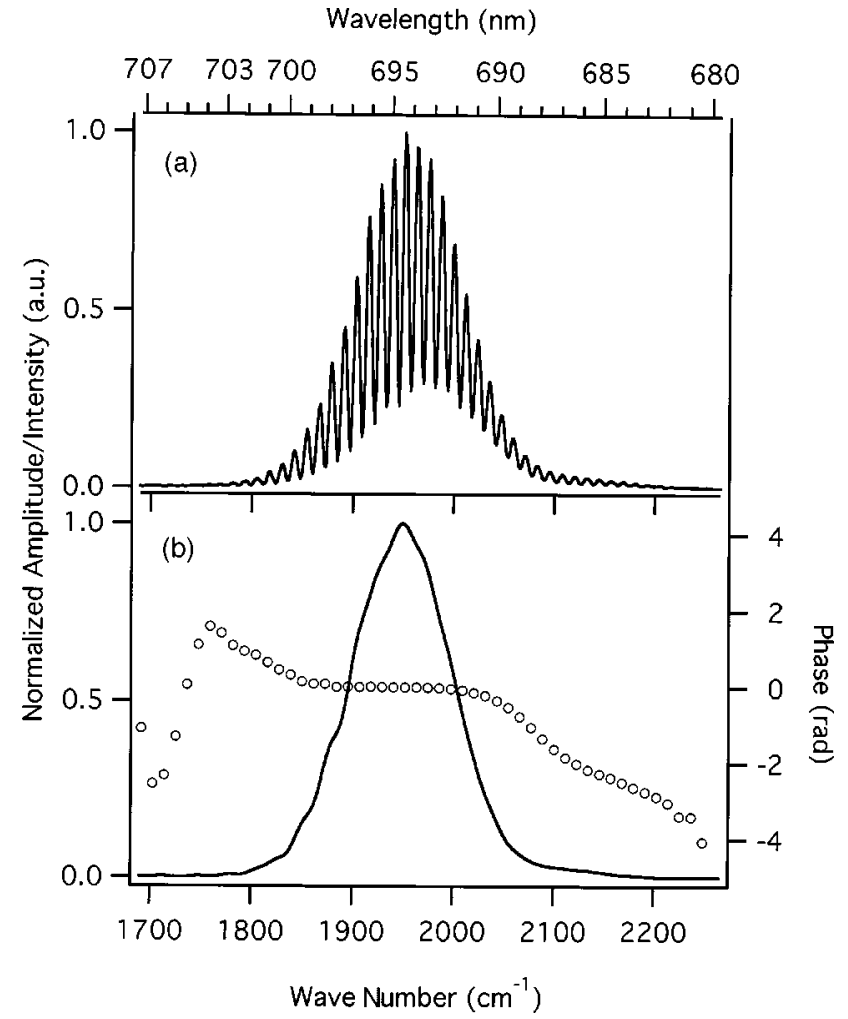

Fig. 3. ZAP SPIDER measurement of the mid-IR pulse. (a) Normalized interferogram and (b) retrieved phase (circles) and normalized amplitude (solid curve).

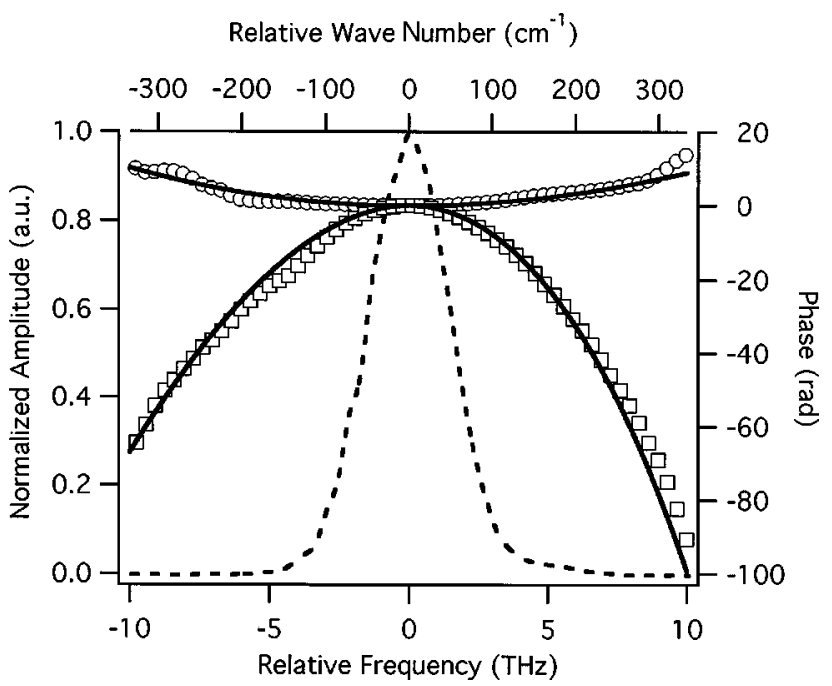

Fig. 4. ZAP SPIDER retrieved phase after transmission through $6.3 \mathrm{~cm}$ of $\mathrm{CaF}_{2}$ (squares) and $6 \mathrm{~mm}$ of Ge (circles) with the calculated phase shown for comparison (solid curves). Normalized amplitude shown for reference (dashed curve). The phase of the unshaped pulse (Fig. 3) has been subtracted from the measured results.

used here has 100 rows of pixels, it is straightforward to measure simultaneously an upconverted mid-IR reference spectrum to record time-resolved, pumpprobe differential absorption spectra. Alternatively, the multiplex advantage gained by upconversion can be used to extend the range of array-detected mid-IR spectra.
One of the distinct advantages of the present upconversion approach is that it can be fully integrated with interference-detected 2D IR spectroscopy based on spectral interferometry. ${ }^{1-7,9}$ The nonlinear signal and a phase-locked external local oscillator are collinearly upconverted with a single chirped 800-nm pulse, yielding a spectral interferogram. Blocking the signal beam and unblocking the second chirped pulse allows the local oscillator to be fully characterized by ZAP SPIDER. Such an implementation also permits the time-domain interferometry required for absolute frequency determination.

Multiplex limitations to the widespread adoption of $2 \mathrm{D}$ vibrational spectroscopy have been addressed by implementing an upconversion method for shifting mid-IR pulses into the visible. Moreover, their amplitude and phase have been measured by an in situ technique that requires only the typically unused zero-order compressor output. The wide transmission bandwidth of $\mathrm{MgO}: \mathrm{LiNbO}_{3}$ allows efficient detection of all fundamental vibrational frequencies above $1820 \mathrm{~cm}^{-1}(\lambda<5.5 \mu \mathrm{m})$, whereas lower frequencies can be measured using other crystals (e.g., $\mathrm{AgGaS}_{2}$ ). Finally, upconversion can be seamlessly integrated into a spectral-interferometry-based implementation of 2D IR spectroscopy with the added capability of in situ ZAP SPIDER characterization of the local oscillator electric field.

This work was partially supported by the U.S. Air Force Office of Scientific Research Multidisciplinary University Research Initiative (grant F49620-02-10381) and the Human Frontier Science Program. K. Kubarych's e-mail address is kevin.kubarych @polytechnique.fr.

\section{References}

1. L. Lepetit and M. Joffre, Opt. Lett. 21, 564 (1996).

2. J. D. Hybl, A. A. Ferro, and D. M. Jonas, J. Chem. Phys. 115, 6606 (2001).

3. M. L. Cowan, J. P. Ogilvie, and R. J. D. Miller, Chem. Phys. Lett. 386, 184 (2004).

4. T. Brixner, T. Mancal, I. V. Stiopkin, and G. R. Fleming, J. Chem. Phys. 121, 4221 (2004).

5. O. Golonzka, M. Khalil, N. Demirdoven, and A. Tokmakoff, Phys. Rev. Lett. 86, 2154 (2001).

6. M. T. Zanni, M. C. Asplund, and R. M. Hochstrasser, J. Chem. Phys. 114, 4579 (2001).

7. N. Belabas and M. Joffre, Opt. Lett. 27, 2043 (2002).

8. P. Hamm, M. Lim, W. F. DeGrado, and R. M. Hochstrasser, J. Chem. Phys. 112, 1907 (2000).

9. M. Khalil, N. Demirdoven, and A. Tokmakoff, Phys. Rev. Lett. 90, 047401 (2003).

10. E. J. Heilweil, Opt. Lett. 14, 551 (1989).

11. P. Baum, S. Lochbrunner, and E. Riedle, Opt. Lett. 29, 210 (2004).

12. P. Baum, S. Lochbrunner, and E. Riedle, Opt. Lett. 29, 1686 (2004).

13. C. Iaconis and I. A. Walmsley, Opt. Lett. 23, 792 (1998).

14. A. C. Dong and W. S. Caughey, Methods Enzymol. 232, 139 (1994).

15. C. Ventalon, J. M. Fraser, and M. Joffre, Opt. Lett. 28, 1826 (2003).

16. P. I. Klocek, Handbook of Infrared Optical Materials (Marcel Dekker, New York, 1991). 